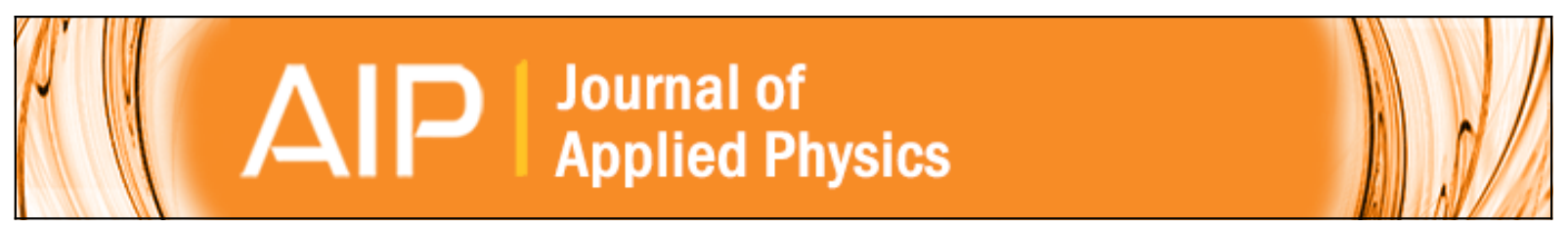

\title{
Acoustically induced transparency using Fano resonant periodic arrays
}

M. Amin, A. Elayouch, M. Farhat, M. Addouche, A. Khelif, and H. Bağcı

Citation: Journal of Applied Physics 118, 164901 (2015); doi: 10.1063/1.4934247

View online: http://dx.doi.org/10.1063/1.4934247

View Table of Contents: http://scitation.aip.org/content/aip/journal/jap/118/16?ver=pdfcov

Published by the AIP Publishing

\section{Articles you may be interested in}

Experimental realization of a variable index transmission line metamaterial as an acoustic leaky-wave antenna Appl. Phys. Lett. 102, 203508 (2013); 10.1063/1.4807280

Phase behaviour and phase separation kinetics measurement using acoustic arrays

Rev. Sci. Instrum. 82, 104902 (2011); 10.1063/1.3650767

Design and implementation of an efficient acoustically levitated drop reactor for in stillo measurements

Rev. Sci. Instrum. 78, 125102 (2007); 10.1063/1.2818798

Transparency induced by coupling of intersubband plasmons in a quantum well

AIP Conf. Proc. 772, 1139 (2005); 10.1063/1.1994512

An acoustic radiometer

Am. J. Phys. 72, 843 (2004); 10.1119/1.1645287

\section{AIP $\mid$ APL Photonics}

APL Photonics is pleased to announce Benjamin Eggleton as its Editor-in-Chief

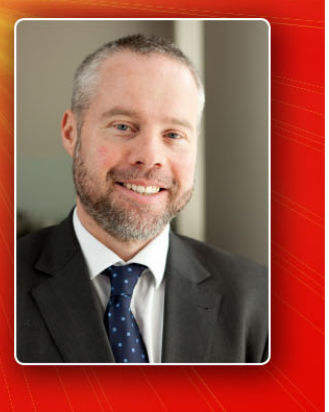




\title{
Acoustically induced transparency using Fano resonant periodic arrays
}

\author{
M. Amin, ${ }^{1, a), b)}$ A. Elayouch, ${ }^{2, b), c)}$ M. Farhat, ${ }^{1, b), d)}$ M. Addouche ${ }^{2}$ A. Khelif, ${ }^{2}$ and H. Bağcı ${ }^{1}$ \\ ${ }^{1}$ Division of Computer, Electrical, and Mathematical Sciences and Engineering, King Abdullah University \\ of Science and Technology (KAUST), Thuwal 23955-6900, Saudi Arabia \\ ${ }^{2}$ Institut FEMTO-ST, Universite de Franche-Comte, CNRS, 15 Bis Avenue des Montboucons, \\ 25030 Besancon, France
}

(Received 27 July 2015; accepted 9 October 2015; published online 22 October 2015)

\begin{abstract}
A three-dimensional acoustic device, which supports Fano resonance and induced transparency in its response to an incident sound wave, is designed and fabricated. These effects are generated from the destructive interference of closely coupled one broad- and one narrow-band acoustic modes. The proposed design ensures excitation and interference of two spectrally close modes by locating a small pipe inside a wider and longer one. Indeed, numerical simulations and experiments demonstrate that this simple-to-fabricate structure can be used to generate Fano resonance as well as acoustically induced transparency with promising applications in sensing, cloaking, and imaging. (C) 2015 AIP Publishing LLC. [http://dx.doi.org/10.1063/1.4934247]
\end{abstract}

\section{INTRODUCTION}

In the recent years, there has been an increasing interest in studying electromagnetic wave propagation in periodic media. ${ }^{1}$ Photonic crystals are among the first engineered periodic structures. Their initial theoretical investigations have almost immediately been followed by their experimental realization. ${ }^{2-7}$ Soon after, focus has been extended to the study of acoustic and elastodynamic wave propagation in periodic media ${ }^{8,9}$ followed by the design and engineering of phononic band gap materials. ${ }^{10-19}$ Many intriguing phenomena have been put forward including negative effective mass density ${ }^{20,21}$ (observed when the acceleration becomes out of phase with the dynamic pressure gradient), negative bulk modulus ${ }^{22}$ (observed when the volume variation is out of phase with the dynamic pressure), and negative acoustic refractive index ${ }^{23-26}$ (observed when both mass density and bulk modulus are negative). Elastodynamic invisibility cloak designs, which make use of the concepts of anisotropic effective mass density and bulk modulus, have been proposed. ${ }^{27-32}$ The equations governing acoustic waves are equivalent to the Maxwell equations in the two-dimensional (2D) case allowing to draw analogies between electromagnetic and acoustic waves. ${ }^{33}$ For example, electromagnetically induced transparency ${ }^{34,35}$ (EIT) and Fano resonance, ${ }^{36,37}$ which are maturing research topics of optics and photonics, could be envisaged in the context of acoustics as well. ${ }^{38,39}$

Resonances with asymmetric Fano lineshapes were originally discovered in the context of quantum mechanics by Fano in 1961. ${ }^{40}$ Quantum Fano resonances are generated from destructive interference of a discrete state with a continuum one. ${ }^{34,41}$ The design of devices capable of supporting Fano resonances is considered as one of the most promising areas of research in the fields of plasmonics and nanophotonics $^{42,43}$ owing to their potential applications ranging from bio-

\footnotetext{
${ }^{\text {a)} E l e c t r o n i c ~ m a i l: ~ m u h a m m a d . a m i n @ k a u s t . e d u . s a ~}$

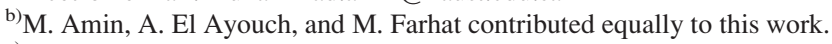

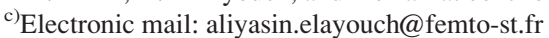

${ }^{\mathrm{d})}$ Electronic mail: mohamed.farhat@kaust.edu.sa
}

chemical sensing, ${ }^{37}$ switching, and filtering to enhancement of nonlinear effects. ${ }^{44}$ On the other hand, acoustic counterparts of Fano resonances and EIT have been surprisingly less investigated. Existing work on the topic has focused on generating acoustically induced transparency (AIT) by coupling modes with highly different $Q$-factors ${ }^{38}$ or by using cancellation of opposite contributions from two detuned acoustic modes $^{39}$ (i.e., equally separated in their spectra but $\pi$-out-of phase). Note that for both of these mechanisms, the term, "mode" refers to the fundamental acoustic harmonic. ${ }^{10}$

In this paper, an acoustic device supporting Fano resonance and induced transparency in its response to an incident sound wave is designed and fabricated. The design consists of a 2D array of subwavelength unit cells, each of which involves a pair of concentric pipes made of a rigid acoustic material (i.e., where $\mathbf{n} \cdot \mathbf{v}=0$, with $\mathbf{n}$ being the vector normal to the surface and $\mathbf{v}$ being the sound wave's velocity, or in terms of pressure $\partial p / \partial n=0$ ). The longitudinal acoustic resonance frequency of a pipe depends on its length and the way it is closed. The frequency of the fundamental resonance in a uniform cylindrical pipe of height $h$ is approximately $c / 4 h .^{45}$ Here, $c$ is the speed of sound. The Fano lineshape and AIT originate from the interference of two different acoustic modes. In the proposed design, this is achieved by locating a small cylinder inside a wider end-capped pipe. Unlike several other acoustic devices previously developed for generating AIT, the design proposed in this work [shown in Fig. 1(a)] is made of true (very-easy to fabricate) threedimensional (3D) coupled resonators, which collectively and effectively act as a phononic crystal when they are arranged in the form of a periodic array [Fig. 1(c)]. This design is more practical than previously proposed waveguide-based ${ }^{39}$ or $2 \mathrm{D}$ structures ${ }^{38}$ since it is easier to fabricate and provides a higher number of degrees of freedom in controlling the amplitude and phase of the sound waves.

The proposed device is fabricated using a $3 \mathrm{D}$ printer, and experiments are carried out using a Kundt's tube. These measurements and numerical simulations, which are carried out using a finite element method, have demonstrated that 


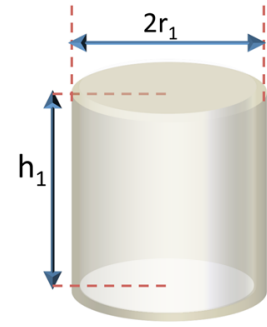

(a)

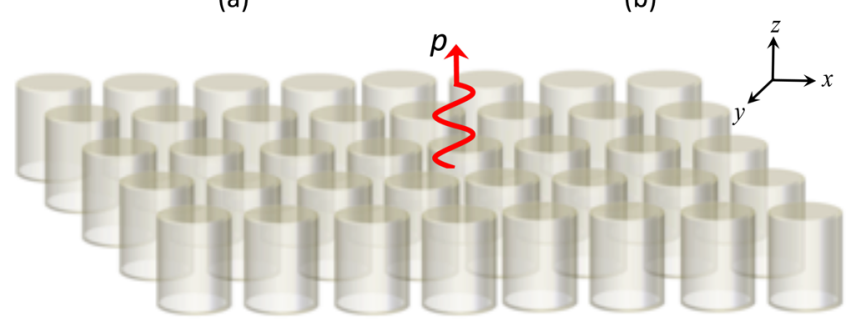

(c)

FIG. 1. (a) Hollow cylindrical pipe of height $h_{1}=20 \mathrm{~mm}$ closed at its top end and open at its bottom. (b) Cross section of the pipe, where outer radius $r_{1}=6 \mathrm{~mm}$ and wall thickness $t=0.3 \mathrm{~mm}$. (c) $2 \mathrm{D}$ periodic array of pipes with cell period $P=15 \mathrm{~mm}$ is excited with a sound wave traveling in the $z$-direction (from bottom to top).

AIT is generated from the destructive interference of two closely coupled but detuned modes in the acoustic response of the proposed design.

\section{RESULTS}

\section{A. Single-pipe resonator}

A hollow pipe made of a rigid acoustic material shown in Fig. 1 supports strong localized resonances when excited by a longitudinal acoustic plane wave (Appendix A). The resonance frequency depends on the pipe's length and its shape. Figures 1(a) and 1(b) show the unit cell of a doubly periodic array that consists of a hollow cylindrical pipe closed at the top end. In these figures and the rest of the text, $h_{1}, r_{1}$, and $t$, and $P$ represent the height, outer radius, and thickness of the (outer) pipe and the cell period of the array, respectively. The acoustic plane wave is normally incident on the periodic array from the bottom as could be seen in Fig. 1(c). It should be noted here that different types and shapes of pipes are possible, but here only cylindrically shaped pipes are considered since they are easier to fabricate using $3 \mathrm{D}$ printers.
Figure 2(a) plots the transmittance (in logarithmic scale) through the array of cylindrical pipes shown in Fig. 1(c). Note that transmittance is defined as $|T|^{2}$, where $|T|$ is the amplitude of the transmitted pressure under the excitation by a unit amplitude plane wave. This figure clearly shows that the spectral lineshape of the mode excited on the array can be described by a symmetric Lorentzian curve, which is considered to be the default lineshape of all interference-free resonances. ${ }^{40}$ The broadband mode induced on this pipe of height $h_{1}$ is labelled as $D_{o}$. This mode resonates around the frequency $f_{o}=c / 4 h_{1} \approx 4 \mathrm{kHz}$ (marked with a circle on Fig. 2(a), where the frequency is normalized by $f_{o}$ in the $x$-axis). Note that the other dimensions of the pipe are given in the caption of Fig. 1. Since the top-end of the pipe is closed, the acoustic pressure field builds longitudinally inside the pipe. Close to the resonance frequency, the scattered pressure increases and starts reflecting the incident pressure fields. At the resonance frequency, the pressure field forms a node at the open end of the pipe and an antinode at the closed one, as could be seen from the pressure field distribution in Fig. 2(b).

Next, the effect of the geometrical parameters on the response of the array to the incident sound wave is investigated. First, the dependence of the transmittance through the array on the height of the pipe $h_{1}$ is characterized. As shown in Fig. 3(a), and as could be expected, a redshift is observed in the resonance frequency as $h_{1}$ is increased while the radius $r_{1}$ and wall thickness $t$ are kept constant. This linear redshift (for example, from $f_{o}$ to $f_{o} / 2$ when $h_{1}$ is doubled) is consistent with the estimated resonance frequency expressed as $c / 4 \alpha h_{1}$. Similarly, the effect of increasing $r_{1}$ and $t$ while $h_{1}$ is kept constant on the transmittance is shown in Fig. 3(b). For a pipe with larger radius and wall thickness, a clear broadening of the resonance width is noted. $Q$-factor changed from 3.8 to 26.2 when the radius and thickness are increased by 1.5 times. Also, a slight redshift is observed in the resonance frequency.

The results in Figs. 3(a) and 3(b) clearly show that interference of two acoustic modes required by Fano resonance and AIT can be obtained using two cylindrical pipes with different sizes. This design is described next.

\section{B. Double-pipe resonator and AIT generation}

The symmetric Lorentzian resonance in Fig. 2(a) is altered to an asymmetric Fano lineshape by inserting a

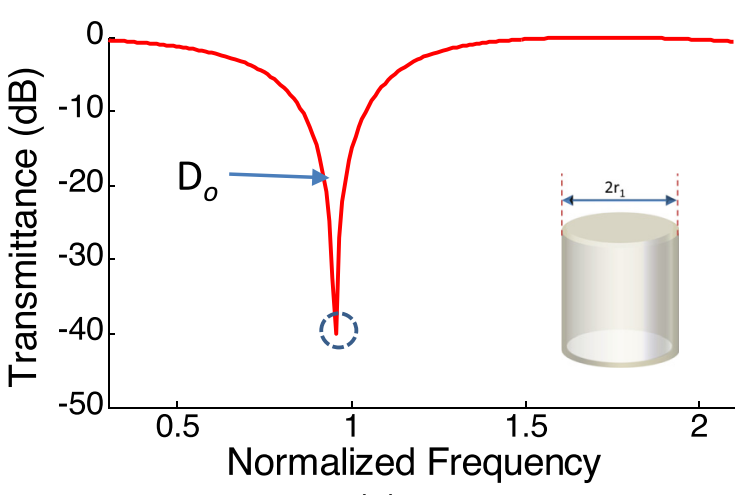

(a)

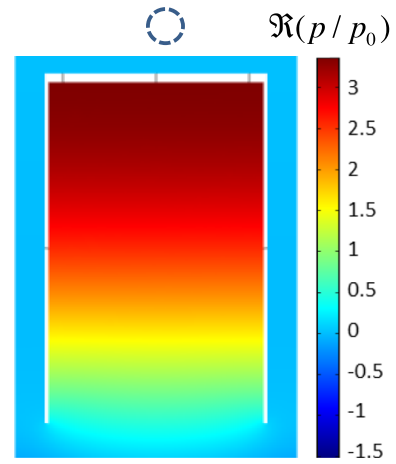

(b)
FIG. 2. (a) Transmittance in logarithmic scale through the array of cylindrical pipes shown in Fig. 1(c). Note that the frequency is normalized by $c / 4 h_{1}$. (b) Real part of pressure field distribution $\left[\Re\left(p / p_{0}\right)\right]$ across a single pipe (normalized to the incident pressure field $p_{0}$ ), at the resonance frequency [marked with a circle on the plot in (a)]. 


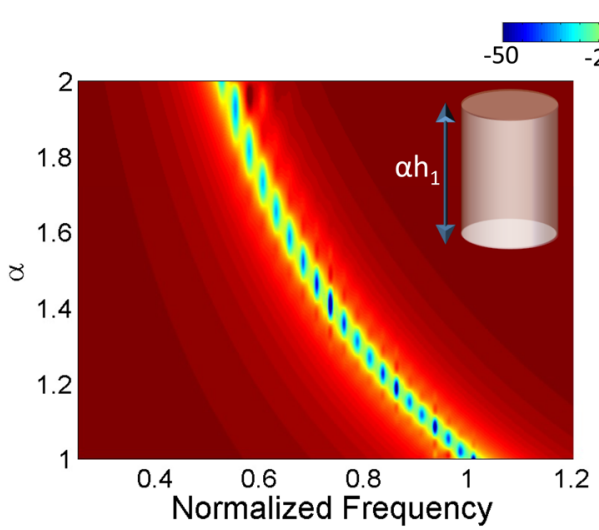

(a)

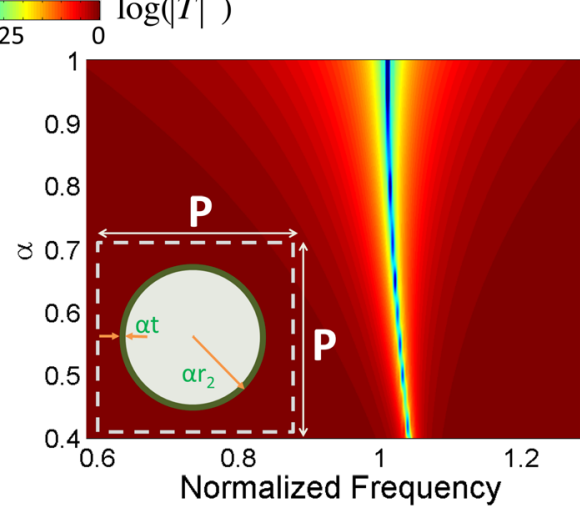

(b)
FIG. 3. (a) Dependence of the transmittance (in logarithmic scale, in $\mathrm{dB}$ ) on the height of the pipe. $h_{1}$ is scaled with $\alpha \in[1,2]$ while the radius of the pipe $r_{1}$ is kept constant. (b) Dependence of the transmittance (in logarithmic scale) on the radius and wall thickness of the pipe. $r_{1}$ and $t$ are scaled with $\alpha \in[0.4,1]$ while height of the pipe $h_{1}$ is kept constant. Note that the frequency is normalized by $c / 4 h_{1}$. second pipe of smaller width and height into the first one as shown in Figs. 4(a) and 4(b). The coupling of the modes associated with each pipe results in generation of a Fano lineshape and AIT in the response of a 2D array of this pipe pair. For this array, the periodicity and dimensions of the outer pipe are the same as those given in Figs. 1(a) and 1(b). The height and outer radius of the inner pipe are $h_{2}=h_{1}-2 t$ and $r_{2}=r_{1}-2 t$, respectively [Figs. 4(a) and 4(b)]. A gap of thickness $t$ between both pipes leaves enough space for the incident acoustic plane wave to couple to the modes induced on both pipes.

On the other hand, the characteristics of the mode associated with the outer pipe change significantly because the acoustic wave interactions on its surface are now limited to a very narrow space within the gap between the two pipes. To isolate this mode, we filled the smaller pipe completely with rigid material as shown in the left inset of Fig. 4(c). Note that this configuration forbids wave propagation in the inner pipe region allowing only the gap mode to be analyzed. Figure 4(c) plots the transmittance through the array of this "blocked" outer pipe. A spectral redshift in the normalized resonance frequency from a value slightly less than 1 to 0.8 is observed.
Additionally, the bandwidth of the mode is significantly reduced; the $Q$-factor is changed from 2.7 to 11.2 . This new shifted and narrower-band gap mode is labelled as $D_{g}$ in Fig. 4(c). On the other hand, the mode associated with the inner pipe alone has a blueshifted normalized resonance at a value slightly higher than 1 , as observed in Fig. 4(c), due to the decrease in the height $\left(h_{2}=h_{1}-2 t\right)$ compared to that of the outer pipe alone. This broadband mode associated with the inner pipe is labelled as $D_{i}$ in Fig. 4(c). Around the resonance peaks, it is well known that the mode fields change their phase. This means that the two detuned modes, $D_{i}$ and $D_{g}$, are out of phase with each other within their respective spectral supports. Therefore, the collective response of the double-pipe resonator exhibits AIT due to destructive interference between the two detuned resonances, with significantly different $Q$-factors. The transmittance within the AIT region is around $60 \%$ [Fig. 4(d)].

The interference characteristics of these longitudinal resonances are exhibited in Fig. 5 showing the pressure field distribution across the center of the pipe at several frequencies marked in Fig. 4(d). At frequency point I, a high pressure field

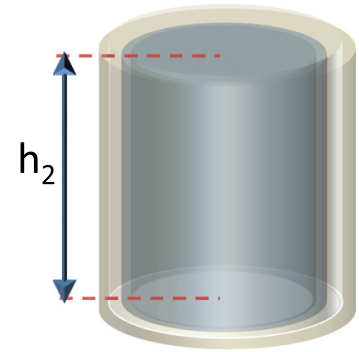

(a)

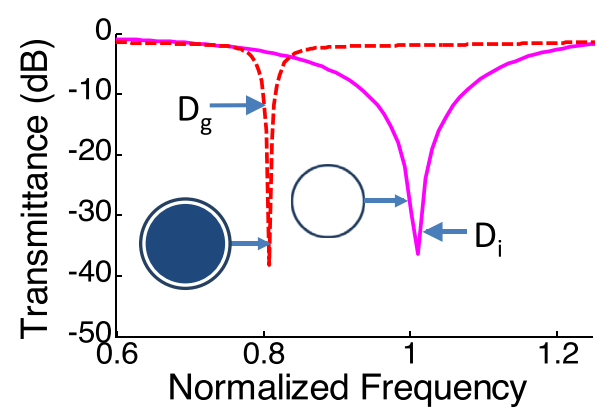

(c)

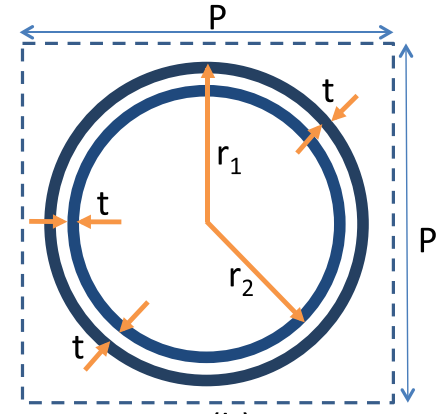

(b)

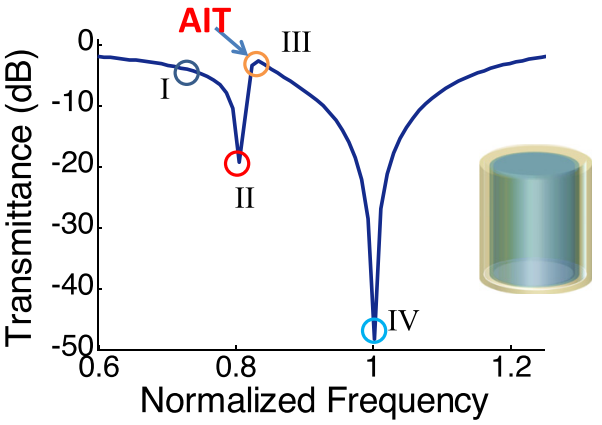

(d)
FIG. 4. (a) Hollow cylindrical pipe of height $h_{2}=h_{1}-t$ closed at its top end and open at its bottom inserted into the pipe described in Fig. 1(a). (b) Cross section of the pipe pair. (c) Transmittance (in logarithmic scale) through the array of double-pipe resonators where inner pipe is blocked (inset on the left) and inner pipe alone (inset on the right). (d) Transmittance (in logarithmic scale) through the array of double-pipe resonators exhibiting asymmetric Fano lineshapes and AIT effect. Note that the frequency is normalized by $c / 4 h_{1}$. 

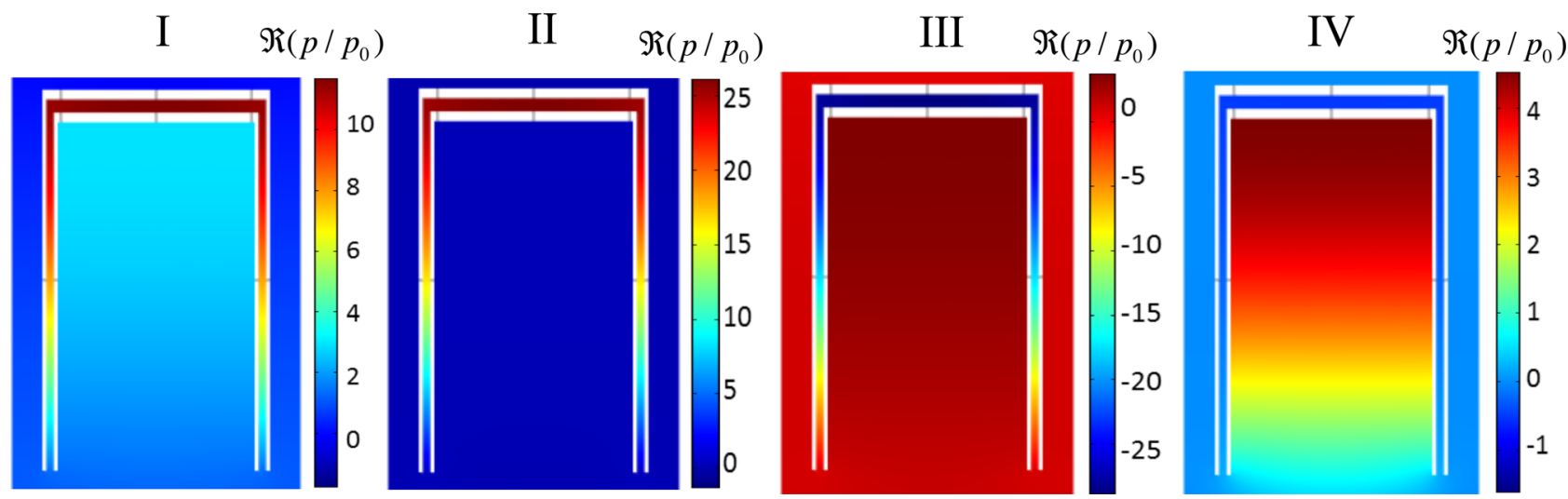

FIG. 5. Real part of the pressure field distribution $\left[\Re\left(p / p_{0}\right)\right]$ across the unit cell (normalized to the incident pressure $\left.p_{0}\right)$ computed at frequency points I, II, III, and IV of Fig. 4(d). The color scales represent the corresponding magnitude of the pressure fields.

develops inside the gap. As point I is far from the resonance peak of $D_{i}$ [Fig. 4(c)], the high pressure fields are confined inside the gap only. Point II is exactly at the resonance frequency peak for $D_{g}$, consequently, high pressure fields are generated inside the gap only (the fields, in dark blue, are zero everywhere else). Also at point II, both pipes interfere constructively (in phase) causing a suppression of transmittance or conversely an enhancement of reflectance, since there is no absorption by the periodic array structure made of a completely rigid material. A sharp increase in transmittance could be noted between frequency points II and III as destructive interference between the two modes takes place. This sharp increase in the acoustic waves transmittance due to destructive interference is attributed to AIT phenomenon. This could be better emphasized by comparing the $Q$-factors of the interfering modes $D_{i}$ and $D_{g}$ in Fig. 4(c). It is interesting to note that between the same two points II and III the sharp increase in transmittance creates a very highly dispersive medium that could be used to generate slow sound. Finally, the frequency point IV corresponds to the resonance peak of $D_{i}$ and therefore the pressure fields are confined within the inner pipe only as shown in Fig. 5. Additionally, the interference in the range between points I and IV in Fig. 4(d) due to the two modes creates a characteristic asymmetric lineshape attributed to Fano resonance.

The geometrical tuning of the double-pipe resonator is also analyzed and its effect on the Fano resonance (and AIT) is demonstrated in Fig. 6. In this case, only the radius $r_{2}$ and the thickness $t$ of the inner pipe are simultaneously tuned. The dimensions of the outer pipe are kept same as in Figs. 1(a) and 1(b). The figure shows that the spectral location and the shape of the acoustic transparency window can be tuned by varying $r_{2}$ : A gradual redshift in the AIT band and a comparatively stronger transmittance at the resonance frequency are observed with an increase in the radius $r_{2}$ and the thickness $t$ of the inner pipe as could be seen in Fig. 6 .

\section{Experimental validation}

The fabricated structure consists of two end-capped pipes that fit into each other, as discussed previously. The smaller pipe is "suspended" inside the wider pipe using two connection points located at the upper sections of the pipes
[Fig. 7(b)]. The dimensions of the structure are: $h_{1}=79.1 \mathrm{~mm}$, $h_{2}=72 \mathrm{~mm}, r_{1}=41.7 \mathrm{~mm}$, and $r_{2}=36 \mathrm{~mm}$, lateral separation (i.e., the lateral gap) is $2.7 \mathrm{~mm}$, depth separation (i.e., the lower gap) $4.05 \mathrm{~mm}$, and thickness of wall $t=3 \mathrm{~mm}$. All is held together by a cylindrical support having an external radius equal to the radius of the Kundt's tube, a thickness of $3 \mathrm{~mm}$, and a height of $79.1 \mathrm{~mm}$. Additionally, another structure consisting of only the inner pipe is fabricated and characterized.

Figure 7(a) plots $20 \log (|T|)$, where $|T|$ is the amplitude of the pressure field transmitted through the single- or double-pipe resonator and is obtained via experiments carried out using the Kundt's tube (Appendix B). In the case of a single pipe [shown in the upper part of Fig. 7(b)], a strong attenuation in the transmittance spectra is observed. Values reaching $-35 \mathrm{~dB}$ around the normalized frequency 1 (corresponding to a frequency of $1100 \mathrm{~Hz}$ ) are obtained. Adding an outer pipe [shown in the lower part of Fig. 7(b)] introduces a new attenuation at the normalized frequency 0.7 (corresponding to a frequency of $760 \mathrm{~Hz}$ ), as well as an AIT effect

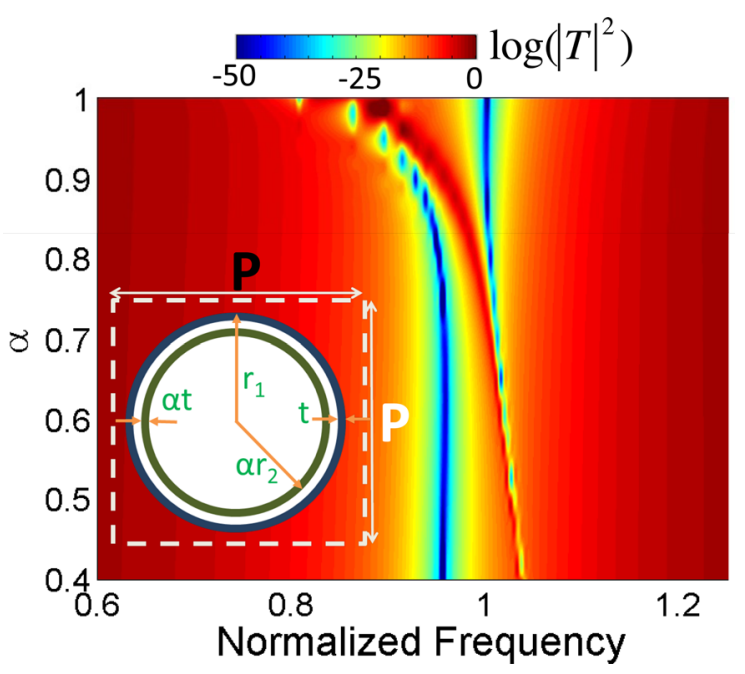

FIG. 6. Effect on transmittance, plotted in logarithmic scale (in $\mathrm{dB}$ ), through the array of double-pipe resonators of varying the radius $\alpha r_{2}$ and the wall thickness $\alpha t$ of the inner pipe, where $\alpha \in[0.4,1]$, while keeping the height $h_{2}$ constant. The other geometrical parameters are same as in Figs. 4(a) and 4(b). The inset shows the schematic view of the corresponding scaling of geometrical parameters. The color scale is given for corresponding amplitudes of transmittance. Note that the frequency is normalized by $c / 4 h_{1}$. 


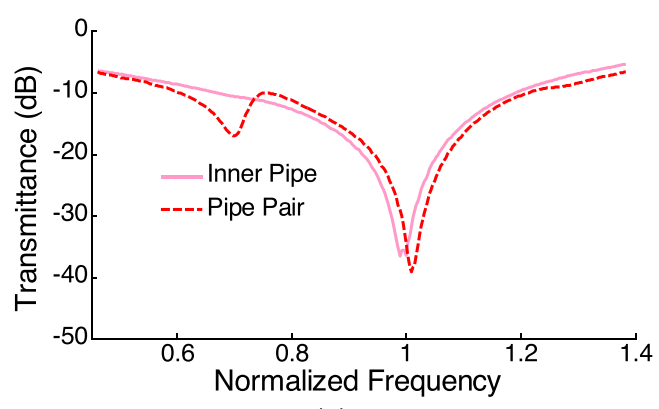

(a)

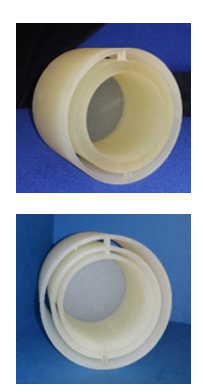

(b)
FIG. 7. (a) Experimentally measured transmittance through the single and double-pipe resonator (insets at the top and bottom, respectively) exhibiting asymmetric Fano lineshapes and AIT effect. Note that the frequency is normalized by $c / 4 h_{1}$. (b) Single (up) and double (bottom) pipe resonator.

that appears between the two resonance frequencies. The shape of the transmittance spectra reveals the signature of a Fano-like resonance, which has been predicted by the numerical simulation plotted in Figs. 4(c) and 4(d).

\section{Enhanced AIT effect}

A closer look to Fig. 7(a) reveals that, within the AIT window, the values of transmittance through the single and double-pipe resonators differ only by a few $\mathrm{dB}$, possibly degrading the effect of AIT. To improve this, the same design with different dimensions is tested. The dimensions of the new structure are: $h_{1}=h_{2}=20 \mathrm{~mm}, r_{1}=12.5 \mathrm{~mm}, r_{2}=6.5 \mathrm{~mm}$, and $t=1 \mathrm{~mm}$. The lateral separations are thus $5 \mathrm{~mm}$ [Fig. 8(b)]. The other parameters of the experimental setup are same as those described in Section IIC. Figure 8(a) plots $20 \log (|T|)$, where $|T|$ is the amplitude of the measured pressure field, which is transmitted through the single- or doublepipe resonator and is obtained via experiments. A strong attenuation is observed in the transmittance spectra of the single-pipe resonator; it reaches $-40 \mathrm{~dB}$ around the normalized frequency 0.977 (corresponding to a frequency $4.19 \mathrm{kHz}$ ). For the double-pipe resonator, around the AIT window, the shape of transmittance spectra is significantly different from that of the single-pipe resonator. The AIT effect appears at the frequency $0.984(4.22 \mathrm{kHz})$, with transmittance reaching $-15 \mathrm{~dB}$, between two dips at $0.956(4.10 \mathrm{kHz})$ and $1.017(4.36 \mathrm{kHz})$. As clearly shown in the figure, the difference between the

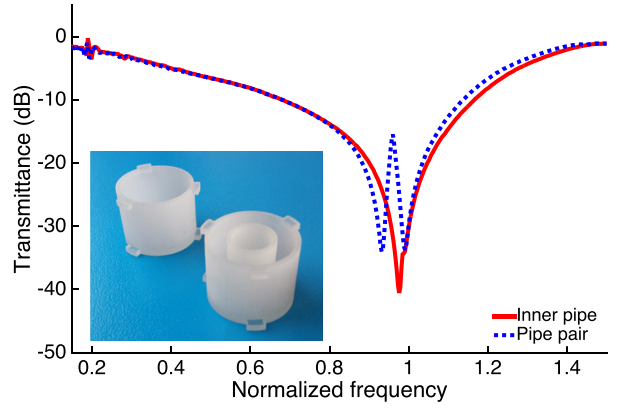

(a)

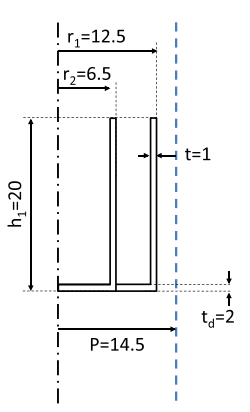

(b)
FIG. 8. (a) Experimentally measured transmittance through the single and double-pipe resonators exhibiting enhanced symmetric Fano lineshapes and AIT effect. (b) Geometry and parameters of the structure (dimensions are in $\mathrm{mm}$ ). transmittance levels of the single and double-pipe resonator is significant, reaching $25 \mathrm{~dB}$.

\section{SUMMARY}

A periodic array of subwavelength sized coupled-pipe resonators, which can support Fano lineshape and AIT in its response to an incident sound wave, is described. The spectral location and lineshape of the Fano resonance and AIT can be tuned by varying the geometrical parameters of the pipes. The proposed design is easy to fabricate; its experimental realization, which is also described in this paper, opens up new possibilities to designing and fabricating novel devices for filtering, imaging, and sensing applications.

\section{APPENDIX A: SIMULATIONS}

In this study, elastodynamic wave interactions are modeled using the Lamé equation. ${ }^{45}$ Due to the physical parameters of our structure, we limit our analysis to longitudinal pressure waves (and ignore transverse shear waves that do not propagate in air). Under the assumption of time harmonic dependence $e^{-i \omega t}$, where $\omega$ is the frequency of excitation, the pressure field satisfies the equation

$$
\nabla \cdot\left(\rho^{-1} \nabla p\right)+\frac{\omega^{2}}{\kappa} p=0 .
$$

Here, $\rho$ is the medium's volumetric mass density, $\kappa$ is its bulk modulus, and $\omega$ is the frequency of the wave. Continuity relations of the pressure $p$ and its flux $\left[\left(1 / \rho_{n}\right) \partial p /\right.$ $\partial n$ ] (proportional to the normal velocity) at the boundaries of the objects, with $\rho_{n}$ the normal component of the density, complete this equation.

It is assumed that the arrays of resonators designed in this work reside in air, and rigid boundary conditions are enforced on the pipe surfaces (absence of vibrations: $\partial p /$ $\partial n=0)$. The density of air and the speed of sound in air are $\rho=1.22 \mathrm{~kg} / \mathrm{m}^{3}$ and $c=343 \mathrm{~ms}^{-1}$, respectively. These material properties are used in the finite element program COMSOL Multiphysics ${ }^{46}$ to carry out the $3 \mathrm{D}$ numerical characterization of acoustic wave interactions on the periodic arrays; the 3D structure is excited by an acoustic pressure plane wave. The excitation is introduced into the unit cell by enforcing a port boundary condition on a transverse reference plane located above the resonator. On the top and bottom of the unit cell, perfectly matched layers (PML) are used as absorbing boundary conditions to "imitate" the behavior of acoustic waves propagating away from the structure. On the side boundaries of the unit cell, periodic boundary conditions are enforced to simulate the effect of an infinite 2D periodic array. The transmitted field is computed on a transverse reference plane located below the resonator.

\section{APPENDIX B: EXPERIMENTS}

The acoustic properties of the structure are studied experimentally using a four-microphone standing wave tube, more commonly referred to as a Kundt's tube. The measurements are carried out using Brüel and Kjæs Transmission 


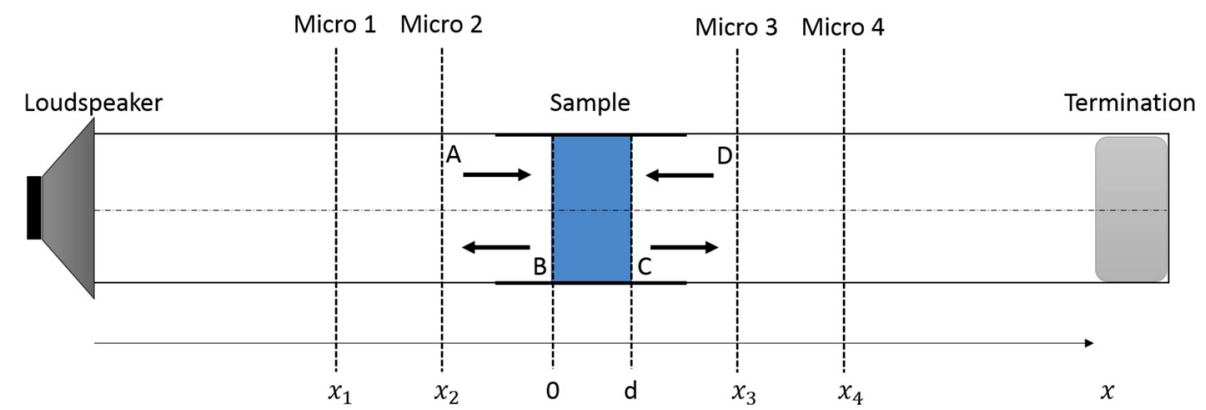

FIG. 9. Schematic view of the standing wave tube with the source, termination, and the structure to be characterized at the center.

Loss Tube Kit Type 4206-T. The sample is "sandwiched" between two parts of the tube, as shown in Fig. 9. The inner diameter of the tube section is $29 \mathrm{~mm}$. A loudspeaker, at one end of the tube, is used for generating a broadband random signal over the frequency range $0.2-6 \mathrm{kHz}$. Acoustic pressure measurements are carried out for two different tube termination conditions, open and approximately anechoic. For each termination condition, complex pressure and velocity fields are computed at each side of the sample material. These values are then used to estimate a two-by-two transfer matrix that characterizes the transmission through the sample. by

In this configuration, transmission coefficient $T$ is given

$$
T=\frac{2 e^{i k d}}{T_{11}+\frac{T_{12}}{\rho_{0} c}+\rho_{0} c T_{21}+T_{22}}
$$

where $T_{11}, T_{12}, T_{21}$, and $T_{22}$ are the entries of the transfer matrix in equation

$$
\left.\left(\begin{array}{cc}
p_{1} & p_{2} \\
v_{1} & v_{2}
\end{array}\right)\right|_{x=0}=\left.\left(\begin{array}{ll}
T_{11} & T_{12} \\
T_{21} & T_{22}
\end{array}\right)\left(\begin{array}{ll}
p_{1} & p_{2} \\
v_{1} & v_{2}
\end{array}\right)\right|_{x=d} .
$$

Here, $\left\{p_{1}, v_{1}\right\}$ and $\left\{p_{2}, v_{2}\right\}$ are pairs of pressure field and the $x$ component of the velocity field associated with the two independent measurements, respectively. They are computed using

$$
\begin{aligned}
& \left.p\right|_{x=0}=A+B \\
& \left.p\right|_{x=d}=C e^{-i k d}+D e^{i k d} \\
& \left.v\right|_{x=0}=\frac{A-B}{\rho_{0} c} \\
& \left.v\right|_{x=d}=\frac{C e^{-i k d}+D e^{i k d}}{\rho_{0} c},
\end{aligned}
$$

where the coefficients $A, B, C$, and $D$ are given by

$$
\begin{aligned}
& A=\sqrt{G_{r r}} \frac{i\left(H_{1 r} e^{i k x_{2}}-H_{2 r} e^{\left.i k x_{1}\right)}\right.}{2 \sin k\left(x_{1}-x_{2}\right)} \\
& C=\sqrt{G_{r r}} i \frac{\left(H_{3 r} e^{i k x_{4}}-H_{4 r} e^{i k x_{3}}\right)}{2 \sin k\left(x_{3}-x_{4}\right)} \\
& B=\sqrt{G_{r r}} \frac{i\left(H_{2 r} e^{-i k x_{1}}-H_{1 r} e^{-i k x_{2}}\right)}{2 \sin k\left(x_{1}-x_{2}\right)} \\
& D=\sqrt{G_{r r}} i \frac{\left(H_{4 r} e^{i k x_{3}}-H_{3 r} e^{i k x_{4}}\right)}{2 \sin k\left(x_{3}-x_{4}\right)} .
\end{aligned}
$$

TABLE I. Characteristics of the Visijet crystal resin used by the 3D printer Projet SD3500.

\begin{tabular}{lccc}
\hline \hline & $\begin{array}{c}\text { Density (liquid) } \\
\text { at } 80(\mathrm{~g} / \mathrm{cm})\end{array}$ & $\begin{array}{c}\text { Young } \\
\text { modulus (MP) }\end{array}$ & $\begin{array}{c}\text { Bending } \\
\text { stress (MP) }\end{array}$ \\
\hline VisiJet crystal resin & 1.02 & 1463 & 49 \\
\hline \hline
\end{tabular}

Here, $H_{i r}$ are the response functions between the complex sound pressures $p_{x_{i}}$ (measured at $x_{i}$ ) and the complex reference signal $r$ provided for the loudspeaker, and $G_{r r}$ is the autospectrum of $r$. It is assumed that $p_{x_{i}}$ can be decomposed as

$$
\begin{aligned}
& p_{x_{1}}=A e^{-i k x_{1}}+B e^{i k x_{1}} \\
& p_{x_{3}}=C e^{-i k x_{3}}+D e^{i k x_{3}} \\
& p_{x_{2}}=A e^{-i k x_{2}}+B e^{i k x_{2}} \\
& p_{x_{4}}=C e^{-i k x_{4}}+D e^{i k x_{4}} .
\end{aligned}
$$

The proposed structure is "fabricated" using the 3D printer Projet SD3500. Characteristics of the resin used by the 3D printer are provided in Table I.

${ }^{1}$ S. Datta, Classical Wave Propagation in Periodic and Random Media (Iowa State University, 1994).

${ }^{2} \mathrm{E}$. Yablonovitch, "Inhibited spontaneous emission in solid-state physics and electronics," Phys. Rev. Lett. 58, 2059 (1987).

${ }^{3}$ S. John, "Strong localization of photons in certain disordered dielectric superlattices," Phys. Rev. Lett. 58, 2486-2489 (1987).

${ }^{4} \mathrm{R}$. Zengerle, "Light propagation in singly and doubly periodic planar waveguides," J. Mod. Opt. 34, 1589-1617 (1987).

${ }^{5}$ T. F. Krauss, R. M. D. L. Rue, and S. Brand, "Two-dimensional photonicbandgap structures operating at near-infrared wavelengths," Nature 383, 699-702 (1996).

${ }^{6}$ B. Gralak, S. Enoch, and G. Tayeb, "Anomalous refractive properties of photonic crystals," J. Opt. Soc. Am. A 17, 1012-1020 (2000).

${ }^{7}$ M. Notomi, "Negative refraction in photonic crystals," Opt. Quantum Electron. 34, 133-143 (2002).

${ }^{8}$ R. V. Craster and S. Guenneau, Acoustic Metamaterials: Negative Refraction, Imaging, Lensing and Cloaking (Springer, Berlin, 2013), Vol. 166.

${ }^{9}$ E. Lheurette, Metamaterials and Wave Control (Wiley-ISTE, London, 2013).

${ }^{10}$ Z. Liu, X. Zhang, Y. Mao, Y. Zhu, Z. Yang, C. Chan, and P. Sheng, "Locally resonant sonic materials," Science 289, 1734-1736 (2000).

${ }^{11}$ C. Poulton, A. Movchan, R. McPhedran, N. Nicorovici, and Y. Antipov, "Eigenvalue problems for doubly periodic elastic structures and phononic band gaps," Proc. R. Soc. London, Ser. A 456, 2543-2559 (2000).

${ }^{12}$ S. Yang, J. Page, Z. Liu, M. Cowan, C. Chan, and P. Sheng, "Focusing of sound in a 3d phononic crystal," Phys. Rev. Lett. 93, 024301 (2004).

${ }^{13}$ L. Feng, X.-P. Liu, Y.-B. Chen, Z.-P. Huang, Y.-W. Mao, Y.-F. Chen, J. $\mathrm{Zi}$, and Y.-Y. Zhu, "Negative refraction of acoustic waves in twodimensional sonic crystals,” Phys. Rev. B 72, 033108 (2005). 
${ }^{14} \mathrm{X}$. Zhang and Z. Liu, "Negative refraction of acoustic waves in twodimensional phononic crystals," Appl. Phys. Lett. 85, 341-343 (2004).

${ }^{15}$ X. Hu, Y. Shen, X. Liu, R. Fu, and J. Zi, "Superlensing effect in liquid surface waves," Phys. Rev. E 69, 030201 (2004).

${ }^{16}$ M. Farhat, S. Guenneau, S. Enoch, G. Tayeb, A. B. Movchan, and N. V. Movchan, "Analytical and numerical analysis of lensing effect for linear surface water waves through a square array of nearly touching rigid square cylinders," Phys. Rev. E 77, 46308 (2008).

${ }^{17}$ M. Farhat, S. Guenneau, S. Enoch, and A. B. Movchan, "All-angle-negative-refraction and ultra-refraction for liquid surface waves in $2 \mathrm{~d}$ phononic crystals," J. Comput. Appl. Math. 234, 2011-2019 (2010).

${ }^{18}$ J. H. Page et al., "Phononic crystals," Phys. Status Solidi B 241, 3454-3462 (2004).

${ }^{19}$ Y. Penneca, J. O. Vasseura, B. Djafari-Rouhania, L. Dobrzynskia, and P. A. Deymier, "Two-dimensional phononic crystals: Examples and applications," Surf. Sci. Rep. 65, 229-291 (2010).

${ }^{20}$ X. Niu, L. Liu, W. Wen, and P. Sheng, "Hybrid approach to highfrequency microfluidic mixing," Phys. Rev. Lett. 97, 044501 (2006).

${ }^{21}$ M. Farhat, S. Guenneau, S. Enoch, and A. B. Movchan, "Negative refraction, surface modes, and superlensing effect via homogenization near resonances for a finite array of split-ring resonators," Phys. Rev. E 80, 046309 (2009).

${ }^{22}$ N. Fang, D. Xi, J. Xu, M. Ambati, W. Srituravanich, C. Sun, and X. Zhang, "Ultrasonic metamaterials with negative modulus," Nat. Mater. 5, 452-456 (2006).

${ }^{23}$ J. Li and C. Chan, "Double-negative acoustic metamaterial," Phys. Rev. E 70, 055602 (2004).

${ }^{24}$ Y. Ding, Z. Liu, C. Qiu, and J. Shi, "Metamaterial with simultaneously negative bulk modulus and mass density," Phys. Rev. Lett. 99, 093904 (2007).

${ }^{25}$ S. Guenneau, A. Movchan, G. Pétursson, and S. A. Ramakrishna, "Acoustic metamaterials for sound focusing and confinement," New J. Phys. 9, 399 (2007).

${ }^{26}$ Z. Liang, T. Feng, S. Lok, F. Liu, K. B. Ng, C. H. Chan, J. Wang, S. Han, S. Lee, and J. Li, "Space-coiling metamaterials with double negativity and conical dispersion," Sci. Rep. 3, 01614 (2013).

${ }^{27}$ G. Dupont, M. Farhat, A. Diatta, S. Guenneau, and S. Enoch, "Numerical analysis of three-dimensional acoustic cloaks and carpets," Wave Motion 48, 483-496 (2011).

${ }^{28}$ M. D. Guild, M. R. Haberman, and A. Alù, "Plasmonic cloaking and scattering cancelation for electromagnetic and acoustic waves," Wave Motion 48, 468-482 (2011).

${ }^{29}$ P.-Y. Chen, M. Farhat, S. Guenneau, S. Enoch, and A. Alù, "Acoustic scattering cancellation via ultrathin pseudo-surface,” Appl. Phys. Lett. 99, 191913 (2011)
${ }^{30}$ A. N. Norris, "Acoustic cloaking theory," Proc. R. Soc. A-Math. Phys. Eng. Sci. 464, 2411-2434 (2008).

${ }^{31} \mathrm{M}$. Farhat, S. Guenneau, and S. Enoch, "Broadband cloaking of bending waves via homogenization of multiply perforated radially symmetric and isotropic thin elastic plates," Phys. Rev. B 85, 020301 (2012).

${ }^{32}$ T. Bückmann, M. Thiel, M. Kadic, R. Schittny, and M. Wegener, "An elasto-mechanical unfeelability cloak made of pentamode metamaterials," Nat. Commun. 15, 4130 (2014).

${ }^{33}$ S. A. Cummer and D. Schurig, "One path to acoustic cloaking," New J. Phys. 9, 45 (2007).

${ }^{34}$ M. Fleischhauer, A. Imamoglu, and J. P. Marangos, "Electromagnetically induced transparency: Optics in coherent media," Rev. Mod. Phys. 77, 633 (2005).

${ }^{35}$ N. Liu, L. Langguth, T. Weiss, J. Kästel, M. Fleischhauer, T. Pfau, and H. Giessen, "Plasmonic analogue of electromagnetically induced transparency at the drude damping limit," Nat. Mater. 8, 758-762 (2009).

${ }^{36}$ B. Luk'yanchuk, N. I. Zheludev, S. A. Maier, N. J. Halas, P. Nordlander, H. Giessen, and C. T. Chong, "The fano resonance in plasmonic nanostructures and metamaterials," Nat. Mater. 9, 707-715 (2010),

${ }^{37}$ C. Wu, A. B. Khscianikaev, R. Adato, N. Arju, A. A. Yanik, H. Altug, and G. Shvets, "Fano-resonant asymmetric metamaterials for ultrasensitive spectroscopy and identification of molecular monolayers," Nat. Mater. 11, 69-75 (2011)

${ }^{38}$ F. Liu, M. Ke, A. Zhang, W. Wen, J. Shi, Z. Liu, and P. Sheng, “Acoustic analog of electromagnetically induced transparency in periodic arrays of square rods," Phys. Rev. E 82, 026601 (2010).

${ }^{39}$ A. Santillán and S. I. Bozhevolnyi, "Acoustic transparency and slow sound using detuned acoustic resonators," Phys. Rev. B 84, 064304 (2011).

${ }^{40} \mathrm{U}$. Fano, "Effects of configuration interaction on intensities and phase shifts," Phys. Rev. 124, 1866 (1961).

${ }^{41}$ C. Ott, A. Kaldun, P. Raith, K. Meyer, M. Laux, J. Evers, C. H. Keitel, C. H. Greene, and T. Pfeifer, "Lorentz meets fano in spectral line shapes: A universal phase and its laser control," Science 340, 716-720 (2013).

${ }^{42}$ A. E. Miroshnichenko, S. Flach, and Y. S. Kivshar, "Fano resonances in nanoscale structures," Rev. Mod. Phys. 82, 2257 (2010).

${ }^{43}$ M. Rahmani, B. Luk'yanchuk, and M. Hong, "Fano resonance in novel plasmonic nanostructures," Laser Photon. Rev. 7, 329-349 (2013).

${ }^{44}$ M. Amin, M. Farhat, and H. Bağc1, "A nonlinear plasmonic resonator for three-state all-optical switching," Opt. Express 22, 6966-6975 (2014).

${ }^{45}$ L. E. Kinsler, A. R. Frey, A. B. Coppens, and J. V. Sanders, Fundamentals of Acoustics (Wiley, New York, 1999), Vol. 1.

${ }^{46} \mathrm{See}$ http://www.comsol.com for Comsol multiphysics (accessed September 10, 2014). 